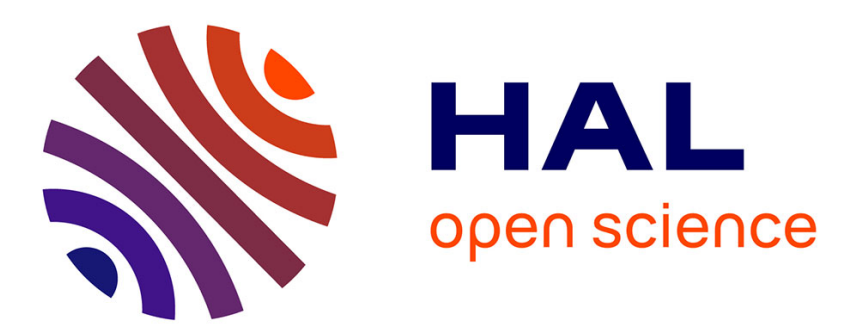

\title{
Les images filmiques et la part insignifiante de l'expérience urbaine
}

\author{
Laure Brayer
}

\section{To cite this version:}

Laure Brayer. Les images filmiques et la part insignifiante de l'expérience urbaine. Multitudes, 2016, 65 (4), pp.184 - 189. 10.3917/mult.065.0184 . hal-01629271

\section{HAL Id: hal-01629271 \\ https://hal.univ-grenoble-alpes.fr/hal-01629271}

Submitted on 6 Nov 2017

HAL is a multi-disciplinary open access archive for the deposit and dissemination of scientific research documents, whether they are published or not. The documents may come from teaching and research institutions in France or abroad, or from public or private research centers.
L'archive ouverte pluridisciplinaire HAL, est destinée au dépôt et à la diffusion de documents scientifiques de niveau recherche, publiés ou non, émanant des établissements d'enseignement et de recherche français ou étrangers, des laboratoires publics ou privés. 


\section{Laure Brayer}

\section{Les images filmiques et la part insignifiante de l'expérience urbaine}

\section{Résumé :}

À partir de The Girl Chewing Gum réalisé par John Smith en 1976, il s'agira ici de rendre compte du travail perceptif et sensible que les images audiovisuelles peuvent induire chez le spectateur. Nous nous intéresserons pour cela à ce qui prend forme au cours de son expérience d'audio-vision, dans l'écart entre images et sons. Il s'agira plus largement d'interroger la capacité des images filmiques à traduire des expériences urbaines sensibles et ordinaires, et d'en favoriser le récit.

\section{Des images pour comprendre et penser la ville contemporaine}

Qu'il s'agisse d'analyser l'espace urbain existant ou de le concevoir, les images détiennent une place importante en architecture tout comme en urbanisme. Au sein des multiples images déployées dans ces champs (plans, cartes, croquis, photographies, etc.), nous proposons ici de porter attention aux images filmiques, dans la mesure où elles nous semblent en capacité de traduire des expériences urbaines sensibles et ordinaires, et d'en favoriser le récit. Cette caractéristique soutient l'hypothèse selon laquelle le film pourrait être opérant pour la conception de la ville en tant que support de débat permettant à différents acteurs de la fabrique urbaine (concepteurs, usagers, élus, techniciens, etc.) de dialoguer ensemble afin de penser collectivement le devenir urbain. La réception filmique, vécue et discutée collectivement, permettrait alors de travailler le sensible et de mettre en partage les représentations de chacun ${ }^{1}$.

Les images filmiques, comme toutes représentations, procèdent d'une sélection, d'une construction de la réalité. Néanmoins, si elles sont loin d'être le «miroir fidèle » (Lefebvre, 1973, cité par Söderström, 2000, p. 16) de l'existant filmé, elles donnent à voir une expérience ordinaire de l'urbain: expérience à la fois banale, quotidienne, mais aussi complexe et sensible. Ainsi, si ces images - au même titre que les images photographiques (Cuny \& Ney, 2013) - se distinguent des autres types de visuels employés en urbanisme, c'est en effet parce qu'elles mettent au travail la perception commune de l'urbain filmé. On peut dire qu'elles rejouent, pour le spectateur, sa perception de la ville, l'amenant à considérer à nouveaux frais la complexité de l'expérience urbaine ordinaire. Révéler la complexité de la ville contemporaine, des pratiques et des usages qui s'y déploient, en appréhendant notamment le caractère métabolique propre à sa transformation ordinaire - les rythmes, les intensités et les nuances d'un lieu filmé -, et mettre à jour et en partage l'importance de la part sensible de notre expérience urbaine ordinaire : telles sont les potentialités des images filmiques quant à la compréhension de la vie urbaine et la conception d'espaces publics urbains ${ }^{2}$.

\footnotetext{
${ }^{1}$ Sur ce dernier point, nous inscrivons notre réflexion à la suite de certains travaux portés par le laboratoire CRESSON et ses collaborateurs, qui travaillent la question du partage des représentations. Voir notamment l'enjeu du transect urbain pour Nicolas Tixier (Tixier, 2011) et le rôle des représentations des espaces du quotidien chez Frédéric Pousin (Pousin, 2012).

${ }_{2}^{2}$ Pour une analyse de la portée du film (comme médium, comme pratique et dans sa réception) dans la perception, la représentation et la conception partagée du paysage urbain et de la transformation ordinaire des lieux, nous nous permettons de renvoyer à notre recherche de doctorat (Brayer, 2014).
} 
À partir de The Girl Chewing Gum réalisé par John Smith en 1976, et notamment au travers de la réception collective d'un extrait de cette production filmique, nous allons, dans le cadre de cet article, tâcher de rendre compte du travail perceptif et sensible que les images audiovisuelles peuvent induire chez le spectateur. Nous nous intéresserons pour cela à ce qui prend forme au cours de son expérience d'audio-vision dans l'écart entre l'image et le son et, puisqu'un commentaire oral se fait ici prédominant sur le plan sonore, entre l'image et la voix off.

\section{De la fille qui mâche de la gomme à la femme derrière la vitre}

The Girl Chewing Gum est le treizième film de John Smith. Inscrit dans le champ du cinéma expérimental britannique, ce réalisateur a commencé à faire des films en 1972, se rapprochant alors du courant structurel-matérialiste et de la coopérative London Filmmakers' Co-op qui rassemblait à cette époque de nombreux cinéastes britanniques d'avant-garde - elle comptait notamment parmi ses membres Peter Gidal, figure emblématique du courant et enseignant de John Smith au Royal College of Art de Londres. Le courant structurelmatérialiste s'est développé en Grande-Bretagne dans les années 1970, dans la lignée du film structurel américain apparu une dizaine d'années auparavant. Alors que le film structurel s'attèle à rendre manifeste le caractère performatif des dispositifs filmiques, la spécificité du film structurel-matérialiste réside dans une «mise au travail» du spectateur. Alain Alcide Sudre précise que «cette doctrine artistique veut que la nature processuelle du film (le "travail") soit mise en avant, afin d'entraver la transparence de la représentation. L'illusion sur laquelle repose la fiction est en effet considérée, par Gidal comme par Le Grice, comme instrument d'aliénation - ruse de l'idéologie dominante. Ainsi, plus que dans le film structurel américain, on insiste ici sur la réflexivité d'une démarche dans laquelle le récepteur ne peut rester dans l'attitude d'admiration passive propre à la catharsis. [...] Caractéristique de l'art conceptuel, l'intention artistique est d'imposer au récepteur une démarche hypothéticodéductive qui le force à partager le travail avec le producteur » (Sudre, p. 340). Comme nous allons le voir, cette intention est particulièrement prégnante dans The Girl Chewing Gum, film dans lequel, à l'instar de la majorité de ses réalisations, John Smith interroge le quotidien avec poésie et humour, réanimant chez le spectateur une curiosité pour les choses banales ou devenues invisibles par habitude. Le spectateur est ainsi mis au travail, et son travail de perception a ici pour terreau l'entre-deux de l'image et du son.

D'une durée de 12 minutes, ce film est composé de deux plans, dont le premier est un plan-séquence de 10 minutes sur lequel nous concentrerons notre attention. Tourné dans l'Est de Londres, à l'angle de Kingsland Road et de Stamford Road, ce premier plan met en scène le quotidien d'une rue animée en début d'après-midi. Des passants déambulant sur les trottoirs et des véhicules empruntant la chaussée traversent le cadre de l'image. Sur le plan sonore, une voix masculine couvre pour partie les sons ambiants. Cette voix off, omniprésente et omnipotente, semble dicter chacun des faits et gestes visibles à l'écran, tel un réalisateur derrière la caméra. La voix précède en effet chacune des actions d'une fraction de seconde : on l'entend ainsi crier «Déplacez lentement la remorque vers la gauche. Et je veux que la fillette traverse en courant, maintenant! ${ }^{3}$, tandis que l'arrière d'un camion disparaît sur la gauche de l'écran et que, sur ce dernier mot, une enfant traverse effectivement le cadre de l'image en gambadant. Mais, après quelques minutes, la voix prend peu à peu du retard - elle ne devance plus chacune des actions vues à l'image - et passe du registre de la prescription à celui de la description, mettant ainsi à jour l'enrayement du dispositif d'énonciation. Lorsqu'elle se met à intimer des ordres à des animaux ou à des éléments du mobilier urbain -

\footnotetext{
${ }^{3}$ Ici comme par la suite, toutes les citations issues du film ont fait l'objet d'une traduction personnelle et apparaîtront en italique.
} 
elle se risque par exemple à apprendre à une horloge la manière dont celle-ci doit dérouler le temps -, le doute du spectateur quant au statut de cette voix s'estompe et laisse place au rire.

Ce film a été réalisé en deux étapes principales. Dans un premier temps John Smith a filmé la rue tandis qu'au même instant un de ses amis procédait à l'enregistrement sonore. Au cours de la captation, certains mouvements de caméra étaient prévus, tandis que d'autres, improvisés, répondaient à ce qui se déroulait dans l'espace public. Ainsi, la caméra filmant depuis la rue réalise des panoramiques verticaux (pour filmer l'horloge fixée sur le toit d'un bâtiment), horizontaux (pour suivre certains passants), et use du zoom. Dans un second temps Smith a rédigé une narration guidée par ce qui avait été enregistré. Les personnages et certains détails de la situation sont des éléments de la réalité filmée qui constituent donc la base de la fiction délivrée par la voix. Par exemple, un bruit strident d'alarme, présent dans l'enregistrement sonore original, est expliqué vers la fin du film, par le commentaire suivant, alors qu'on voit un homme s'éloigner, les mains dans les poches : "Ce jeune homme vient de braquer la poste du quartier, et il s'efforce de passer inaperçu. Il essaie de rester calme, mais sa main transpire alors qu'il serre la crosse du révolver encore plus fort dans la poche droite de son manteau ».

Smith propose ainsi une fictionnalisation du réel et met au travail le spectateur en s'appuyant sur la confusion possible concernant le statut off ou hors-champ de la voix. Si, dans le cadre du cinéma, ces deux types de sons font partie des sons acousmatiques - c'est-àdire dont la source n'est pas visible à l'image -, ils se distinguent néanmoins selon qu'ils s'inscrivent ou non dans le cours de l'action, au cœur de la situation filmée : pour Michel Chion, le son "hors-champ" est «seulement celui dont la cause n'est pas visible simultanément dans l'image, mais qui reste pour nous situé imaginairement dans le même temps que l'action montrée, et dans un espace contigu à celui que montre le champ de l'image ", tandis que le son "off" est "seulement celui qui émane d'une source invisible située dans un autre temps et/ou un autre lieu que l'action montrée dans l'image (musique de film, voix-off du narrateur racontant l'action au passé)»(Chion, 1985, p. 31 et 32). Cette possible confusion entre une voix qui provoque et une voix qui décrit, s'appuyant sur l'espace implicite du hors-champ, constitue ainsi le ressort comique de ce petit drame.

Cependant, outre l'humour, que perçoit-on en tant que spectateur au travers de cette description? Que nous est-il donné à comprendre dans l'entre-deux de l'image et du commentaire, dans cet espace interstitiel dessiné par l'articulation du champ et de la parole ?

\section{Flux de la voix et perception du rythme de la rue}

Le dispositif relatif à la dimension énonciative à l'œuvre dans The Girl Chewing Gum amène sous une forme originale à percevoir l'ambiance du lieu filmé. C'est d'abord par la description exhaustive à laquelle la voix s'exerce que le spectateur est invité à percevoir la rue comme un cadre de vie où prennent place diverses personnes et activités. Le commentaire présente ainsi les passants (leurs genres, âges, origines, relations de parenté, habillements, activités professionnelles, etc.), leurs gestes et attitudes ("regardez devant vous", "montrez du doigt", "se frotte les yeux", "croisez les bras", "regardez à gauche et à droite en traversant", "ils bavarderont ensemble", etc.), mais aussi les manières dont ils marchent, les trajectoires et les vitesses de leurs déplacements ("traversez de gauche à droite", "en courant", "traverser prudemment", "la fille qui mâche du chewing-gum apparaît", "arrêtez-vous", "repartez", etc.). Les propos de Smith décrivent encore les véhicules mobiles ainsi que le cadre bâti ("la remorque", "la rue", "l’horloge", "la fenêtre", "la vitre", "le cinéma", "la banque", "le van").

Selon les catégories d'ancrage et de relais énoncées par Roland Barthes pour distinguer deux fonctions possibles du message linguistique par rapport au message iconique, nous pouvons considérer que le commentaire déployé dans The Girl Chewing Gum prend la fonction d'ancrage. En nommant ce qui est vu à l'image, la parole ancrage aide le spectateur à 
« choisir le bon niveau de perception ; elle [lui] permet d'accommoder non seulement [son] regard, mais encore [son] intellection » (Barthes, 1952, p. 31). Ainsi la parole ancrage dirige l'attention du spectateur, contrôle sa lecture de l'image « qui détient la charge informative, et, comme l'image est analogique, l'information est en quelque sorte plus "paresseuse" » (ibid., p. $33)$.

Cependant, le qualificatif de « paresseux » ne semble pas complètement coïncider avec l'expérience d'audio-vision que l'on peut faire de The Girl Chewing Gum. Si, dans le premier temps de la projection, le spectateur perçoit ce qui se passe à l'image en fonction de ce qui est guidé par le commentaire, c'est justement le décrochage progressif de cette parole qui donne à percevoir autre chose à l'image. D'une part, outre la qualification des présences et de leurs attitudes au moment du tournage, et au-delà des entités décrites par le commentaire, c'est l'articulation de la description même, son flux, qui donnent à percevoir au spectateur le rythme de la rue. À travers l'accélération du débit de la voix ou les pauses marquées dans le cours du récit, c'est en fait l'affluence des passants ou leur absence qui se révèle, c'est le tempo du lieu qui apparaît en filigrane. Une lecture possible du rythme de l'ambiance se dégage ainsi entre l'image et la voix. Et d'autre part, c'est ce qui n'est pas commenté qui se révèle progressivement, le spectateur cherchant alors dans l'image ce qui n'est pas décrit par la voix. Dès lors, si le travail perceptif du spectateur se situe entre l'image et le son (et plus particulièrement dans le cas de ce film, entre l'image et le commentaire parlé), le travail du sensible se situe en-deçà du langage.

\section{Rencontre et constitution d'une communauté sensible à l'orée de la parole}

Selon Pierre-Damien Huyghe, il y a dans certains films, «à un moment donné, la rencontre étonnante de quelque chose qui, sans divertir mon attention, distrait mon esprit, un "à-coté" dont la vertu est de faire incidence, sinon même incident, dans la trame signifiante et dramatisante que constitue inévitablement un film en tant qu'il est aussi fait, matériellement, d'une succession de plans "montés" » (Huyghe, 2012, p. 141). Il y a donc des moments où le spectateur rencontre quelque chose d'insignifiant qui néanmoins le trouble. Si ces moments, que Pierre-Damien Huyghe qualifie de "moments de cinéma », existent pour le spectateur lors du visionnage, nous pensons qu'il peut aussi se passer quelque chose de l'ordre du moment dans le partage des expériences spectatorielles, c'est-à-dire au cours de la discussion, par le commentaire qui prolonge une réception filmique collective. Ces moments s'articulent alors en quelque sorte autour de la rencontre de rencontres : lorsque ce qui a fait trouble chez un spectateur fait écho à l'expérience d'un second; lorsque ces rencontres individuelles se rencontrent dans la discussion et s'exposent au collectif.

Un tel moment s'est produit lors de la discussion d'un extrait ${ }^{4} \mathrm{du}$ film The Girl Chewing Gum projeté au cours d'un entretien collectif basé sur une expérience d'audio-vision partagée - une dizaine de personnes ${ }^{5}$, réunies autour de la projection d'extraits de films, étaient invitées à décrire ce qui importait dans leur réception filmique et à échanger leur compréhension des lieux filmés à partir des fragments qui leur étaient proposées. Dans cet extrait, si la voix omniprésente du narrateur dit beaucoup, elle ne dit cependant pas tout ce que l'on voit à l'image. Décrivant ainsi les présences, les passages et les actions des usagers de la rue, la voix ne fait néanmoins pas mention de la silhouette d'une femme située derrière la fenêtre du bâtiment qui compose l'arrière-plan de l'image. Le verre brouillé de la vitre derrière laquelle elle se tient, la faible portée de ses mouvements et le fait qu'elle soit mise à l'écart du commentaire rendent sa présence quelque peu fantomatique, du moins énigmatique. Il s'agit d'une présence insignifiante, difficilement remarquable, qui pourtant trouble le spectateur. Cet à-côté de la scène, cette autre chose qui n'est pas l'objet du film mais toutefois

\footnotetext{
${ }^{4}$ Il s'agissait d'un court extrait situé au début du film : de 0 à 2 min. et $40 \mathrm{sec}$.

${ }^{5}$ Issus des milieux universitaires et opérationnels, spécialistes ou familiers de la thématique «ville et film ».
} 
le trouble, l'opacifie, le complexifie par sa présence, a suscité des commentaires de la part de différents spectateurs. On peut dire que dans la discussion, il y a alors eu rencontre des rencontres de chaque spectateur à la présence énigmatique.

Ces moments de rencontre, lorsqu'ils arrivent, sont l'expression d'une parole collective travaillant le sensible. Dans ces moments, les propos individuels se réunissent pour accorder de l'importance à l'insignifiant, à ce qui ne fait pas l'objet de la représentation mais s'y invite, $\mathrm{y}$ siège et marque l'expérience du spectateur. Ces moments opèrent ainsi le passage d'un trouble individuel à un trouble partagé qui compte pour le collectif. Il se joue alors une percée du sensible porté par le film, qui, bien qu'insignifiant - qui ne fait pas sens -, importe et demande à être commenté et partagé collectivement. Si la discussion à l'issue d'une projection ne forge pas complètement le sens de l'objet du trouble, elle permet, du moins, de donner de l'importance au registre sensible de l'expérience. Lors des moments de rencontres dont nous parlons, le film contribue alors à établir une communauté sensible.

\section{L'importance de l'insignifiant dans l'expérience urbaine}

Nous pensons ainsi que les images filmiques ont cette capacité à faire surgir et à mettre en débat les formes sensibles de l'expérience urbaine qui relèvent souvent de l'ordre de l'insignifiant : une sensation, une lumière qui se reflète sur une surface bâtie, un mouvement favorisé par la déclivité d'un sol, une manière de percevoir et d'être avec l'autre, de partager ou non un espace et un moment, etc. Ce qui fait qu' « un lieu nous affecte par sa faille intrinsèque, par le flottement du sens qu'il engendre, par l'énigme qui l'habite » (Mons, 2003, p. 2), ce qui donc ne fait pas directement sens mais qui nous meut et nous émeut : tout cela est au cœur de l'expérience urbaine ordinaire.

Appréhender la ville et penser sa conception à partir des images filmiques nous amène ainsi à reconnaître l'importance du sensible dans l'expérience urbaine ordinaire. Il s'agit dès lors de se donner les moyens d'être sensible à ce qui arrive, à ce qui se module dans l'existant, et d'accepter avec légèreté de laisser ouvertes des possibilités d' " accidents », de rencontres, de surgissement du sensible dans l'espace que l'on conçoit.

\section{Bibliographie}

BARTHES Roland. «Rhétorique de l'image ». L'obvie et l'obtus, Essais critiques III. Paris : Seuil, 1982. Pages 25-42. Collection Tel Quel, dirigée par Philippe Solers.

BRAYER Laure. Dispositifs filmiques et paysage urbain : La transformation ordinaire des lieux à travers le film. Thèse de doctorat en architecture. Grenoble : Université de Grenoble, 2014. 510 p. [En ligne]. URL : http://hal.univ-grenoble-alpes.fr/tel-01123772 (consulté le 01/10/2016).

CHION Michel. Le son au cinéma. Paris : Cahiers du cinéma, Éditions de l'étoile, 1985. 220p. Collection Essais, dirigée par Alain Bergala et Jean Narboni.

CUNY Cécile \& NEZ Héloïse. La photographie et le film : des instruments de pouvoir ambivalents. Participations. Revue de sciences sociales sur la démocratie et la citoyenneté. CUNY Cécile \& NEZ Héloïse (Eds.). $\mathrm{N}^{\circ} 7$ : «Photographie et film : antidotes à la domination politique ?», 2013/3. Bruxelles : Éditions De Boeck Supérieur, 2013. Pages 7-46.

HUYGHE Pierre-Damien. Le cinéma avant après. Grenoble : De l'incidence Éditeur, 2012. 221 p.

MONS Alain. L’intervalle des lieux. Le Portique. $\mathrm{N}^{\circ} 12$ : «Charme et séduction », 2003. [En ligne]. Mis en ligne le 15 juin 2006. URL : http://leportique.revues.org/578, (Consulté le 01/10/2016). $8 \mathrm{p}$.

POUSIN Frédéric. « Le quotidien et les ambiances : histoires croisées ». In THIBAUD Jean-Paul \& SIRET Daniel (Eds.). Ambiances en acte(s) / Actes du $2^{\text {nd }}$ Congrès International sur les Ambiances. Grenoble : Réseau International Ambiances, ENSAG, 2012. Pages 331-336.

SMITH John. The Girl Chewing Gum. 12 min. Noir \& blanc, sonore, 1976. [En ligne]. URL : http://www.derives.tv/The-Girl-Chewing-Gum, (Consulté le 01/10/2016). 
SÖDERSTRÖM Ola. Des images pour agir : Le visuel en urbanisme. Lausanne : Éditions Payot Lausanne, 2000. $139 \mathrm{p}$.

SUDRE Alain Alcide. "Expérimental (film)». In VIRMAUX Alain \& VIRMAUX Odette (Eds.). Dictionnaire du cinéma mondial. Mouvements, écoles, courants, tendances et genres. Monaco : Éditions du Rocher, 1994. Pages 318-345.

TIXIER Nicolas (Direction scientifique) \& alii. L'ambiance est dans l'air: La dimension atmosphérique des ambiances architecturales et urbaines dans les approches environnementalistes. Contrat de recherche PIR Ville et Environnement - CNRS - PUCA, novembre 2008- avril 2010. Grenoble : Cresson, ENSAG, 2011. 254 p. 